\title{
Effect of Gabapentin on postoperative pain control after a cesarean delivery: A pilot randomized controlled trial
}

\author{
Tienne Randolf ${ }^{1}$, Cynthia Evrard $^{1}$, Harini Gurram ${ }^{1}$, David M Haas ${ }^{1 *}$, Amy Dorwart ${ }^{2}$ and Nicole Ngo $^{3}$ \\ ${ }^{1}$ Department of Obstetrics and Gynecology, Indiana University School of Medicine, USA \\ ${ }^{2}$ Department of Anesthesia, Indiana University School of Medicine, USA \\ ${ }^{3}$ Investigational Pharmacy at Sidney and Lois Eskenazi Hospital, Indianapolis, USA
}

\begin{abstract}
Background: Opioid dependence has risen dramatically and is becoming more common in pregnant women. Finding adjunct methods to reduce the need for postoperative opioid pain medications is needed. The objective of this pilot trial was to evaluate if administration of gabapentin after cesarean delivery would decrease postoperative opiate requirements and pain scores.

Methods: This was a pilot, triple-blind, placebo-controlled, randomized trial. Women undergoing a non-emergent repeat cesarean delivery who only planned to bottle feed their infants were recruited. Gabapentin $(600 \mathrm{mg})$ or placebo was administered every 8 hours for the first 48 hours after a cesarean delivery. The primary outcome was opiate consumption in the acute postoperative period. All pain medications received during that time frame were converted to morphine equivalents for statistical analysis. The secondary outcomes assessed included pain scores and adverse events.

Results: Over 15 months, 327 women were screened, 33 were found eligible, 13 women were consented and randomized as follows: 3 to gabapentin, 10 to placebo. One woman was withdrawn after having to be converted to general anesthesia during her cesarean delivery. There was a significant reduction in total morphine equivalent consumption at 48 hours (placebo vs. gabapentin: total during first 48 hours- $22.6 \pm 14.1$ vs. $8.9 \pm 2.5$, $p=0.02$ ). The mean pain scores at rest and with movement had no statistically significant differences. There was also no difference in the side effects experienced between the two groups.
\end{abstract}

Conclusion: In this small pilot study, the use of gabapentin in the acute postoperative period following a cesarean delivery appears to reduce opiate consumption without significantly altering VAS pain scores or incidence of adverse side effects.

Abbreviations: EPDS: Edinburgh Perinatal Depression Scale/ Screening; ID: Identification; NIH LACTMED: National Institutes of Health Drugs and Lactations Database.

\section{Introduction}

Opiate dependence in women of reproductive age is a concerning rising epidemic. Death rates of women from prescription opiate abuse is on the rise [1]. One of the most vulnerable times for women to be exposed to prescription opiates is in the postpartum period, particularly in the acute postoperative period after a cesarean delivery [2]. Higher proportions of prescription opiate abuse are observed in women with a history of a cesarean delivery, a procedure performed over one million times annually $[3,4]$. Optimal management of postoperative cesarean pain is important for both maternal and infant health [5,6]. Currently, postoperative cesarean pain is managed with neuraxial anesthesia, non-steroidal anti-inflammatory drugs, opioids, and acetaminophen. However, there is a significant subset of patients for which these therapies do not adequately relieve pain [7]. Under-management of acute postoperative cesarean pain has been associated with breast feeding difficulties, chronic pain, and postpartum depression $[5,6]$. Given these trends, evaluating methods to adequately treat acute postoperative pain cesarean pain is pivotal. Currently, gabapentin is utilized in other perioperative surgical settings to reduce the amount of opioid pain medication required after surgery and to decrease pain sensitization [8].
Gabapentin was approved in 1993 as an anti-epileptic drug [8]. By the late 1990s it became a first line treatment for patients with chronic neuropathic pain [7,8]. Currently, gabapentin is being used as an adjunctive treatment for acute and chronic post-surgical pain [7-9]. In non-obstetrical abdominal procedures, it has been demonstrated that gabapentin is a safe and effective drug for treatment of acute postoperative pain. $[7,8,10]$ In addition to reducing the amount of opioid consumption in the acute postoperative period, it has also shown promise in the reduction of chronic postsurgical pain $[5,8]$. The mechanism of postoperative pain involves numerous neurophysiologic and neurochemical mechanisms $[8,11,12]$. The proposed antihyperalgesia mechanism of action of gabapentin is its ability to bind to the $a 2 \delta$ subunit of voltage-dependent calcium channels in pain transmitting neurons $[8,9]$. Through this modulation, gabapentin may inhibit pain transmission and central sensitization. Gabapentin is well tolerated and the most common side effects are sedation, dizziness, nausea and visual disturbances [8]. Rare side effects include peripheral edema and potential suicidal behaviors.

${ }^{*}$ Correspondence to: David Haas, Department of Obstetrics and Gynecology, Indiana University, 550 University Blvd Rm 2440, Indianapolis, IN 46202; Tel:(317) 880-3960; USA, E-mail: dahaas@iupui.edu

Key words: gabapentin, cesarean, postoperative pain, pilot randomized trial

Received: August 10, 2019; Accepted: September 10, 2019; Published: September 20, 2019 
Currently, there are only a few studies that have looked at the use of gabapentin in the perioperative period to improve cesarean delivery analgesia, but the results are conflicting and further investigation is warranted [13-15]. The objective of this pilot trial was to evaluate if administration of gabapentin after a cesarean delivery would decrease postoperative opiate requirements and pain scores.

\section{Methods}

This was a pilot, triple-blind, placebo-controlled, randomized trial. The study was approved by the institutional review board of Indiana University (Indianapolis Indiana) and was registered on clinicaltrials. gov (NCT\#02490345). CONSORT guidelines were utilized in the design and conduct of the trial.

Women were eligible if they were undergoing a non-emergent cesarean delivery, were at least 18 years of age, and were at least 30 -weeks' gestation. This gestational age was chosen to reduce the likelihood of an intraoperative decision to perform a classical cesarean delivery. Spinal anesthesia or a combined spinal-epidural was utilized during the cesarean deliveries for surgical anesthesia. Participants were excluded from the study if they had: a history of opiate abuse or were currently on opiates during the pregnancy; preexisting chronic pain syndrome; history of depression requiring medication management; required general anesthesia for cesarean delivery; planned classical cesarean delivery; required treatment with magnesium sulfate in the postoperative period; or planned to breastfeed their baby. Although the data on infant exposure to gabapentin through breast milk have not revealed adverse infant effects, women who desired to breastfeed were excluded from this pilot study because the studies are small and limited.

Randomization was performed centrally by the Eskenazi Health Inpatient Investigational Pharmacy using a computer-generated random number sequence. Allocation was concealed by having the pharmacy place the study drug into sequentially numbered envelopes that were provided. When a woman consented to the study, the Investigational Pharmacy was contacted and the next allocation in sequence was assigned to the consented subject. All gabapentin and placebo starch capsules were identically over-encapsulated to provide for blinding of participant, provider, and outcomes assessor. Only the Investigational Pharmacy knew which subjects were assigned to gabapentin or placebo and the code was broken only at the final analysis. There was also a condition for the code to be broken if there was a potential safety signal at any blinded safety analyses, however this never occurred.

Women who were admitted and consented for a non-emergent, scheduled cesarean delivery were evaluated for eligibility. As part of the screening process, the participants were given an Edinburgh Perinatal Depression Screening (EPDS) assessment at the time of consent unless one had already been administered recently during the pregnancy. A score of 10 or more was noteworthy and the Principal Investigator determined if the subject would be excluded. If question \#10 ("The thought of harming myself has occurred to me...") on the screening tool was either "Yes", "Quite often", or "Sometimes", the subject was excluded and a mental health provider would have been contacted to assess the woman in the hospital. If the woman was found to be eligible, informed consent was obtained by the research team

After a participant consented, the entire surgical care team was notified of her participation in the trial. Additionally, the investigational pharmacy was given her information for creation of a study ID and randomization. The anesthesia team for the cesarean delivery was notified and used a predetermined regional anesthesia protocol. The intrathecal dose of opioid was standardized (Fentanyl $25 \mathrm{mcg}$ and Morphine $0.2 \mathrm{mg}$ ), while the amount of intrathecal and epidural local anesthetic was determined based on height and weight of the woman.

In the recovery room after completion of the cesarean delivery, the study drug envelope from the Investigational Pharmacy was opened, and the care nurse administered the study drug capsule to the study participant. In prior studies there were a wide range of postoperative gabapentin dosing durations and regimens and no studies defining the optimal postoperative treatment duration [8]. The elimination half-life of gabapentin is between five and nine hours, and as a result it generally requires dosing three times daily [7]. In the chronic pain setting, a starting dose of $300 \mathrm{mg}$ every eight hours is typically used to avoid unwanted side effects of sedation and dizziness [10]. This dose can then be titrated up to the desired therapeutic dose, which can be up to $3600 \mathrm{mg}$ daily $[10,11]$. Doses of $600-1200 \mathrm{mg}$ over a short period of time have been used and proven to be effective postoperatively with minimal adverse side effects [16]. One study of the dose response of gabapentin on postoperative pain found that doses of $600 \mathrm{mg}$ provided the best balance between pain relief and unwanted side effects [16]. Since then, additional studies have shown benefit in using gabapentin doses of $600 \mathrm{mg}$ or higher in post-surgical patients with minimal adverse effects $[8,11,12]$. Thus, this was the dose chosen for this pilot study. Women in this pilot study were assigned to take study capsules (either $600 \mathrm{mg}$ gabapentin or placebo) every 8 hours for the first 48 hours after the cesarean delivery. Participants were allowed to withdraw from the study at any time. Women in the study were able to ask for as many opioid and non-opioid pain medications as they needed to control their pain during the hospitalization.

Postoperative pain was assessed on a Visual Analog Scale by the care nurses or study personnel before each dose of study drug, before receiving any opioid pain medication, 2-4 hours after study drugs were consumed, at 24,36 , and 48 hours post-operatively, and at any other times during routine postoperative clinical care warranted. Pain scores at 24, 36, and 48 hours were asked at rest and with activity. An EPDS was administered to the subject at approximately 48 hours following delivery, but up to 72 hours after delivery was acceptable. Prescription opioids were given per clinical routine care. Combination hydrocodone $5 \mathrm{mg} /$ acetaminophen $325 \mathrm{mg}$ tablets were the prescribed opioid analgesic after the cesarean deliveries. These were typically prescribed with the ability to take 1 or 2 pills every 6 hours. Women also received $800 \mathrm{mg}$ ibuprofen every 8 hours. Study personnel noted the times and amount of opiate medications utilized by participants in the hospital and how many pills participants received upon discharge. One week (6-9 days) after the cesarean delivery, participants were contacted by phone or other preferred method. Information regarding pain, nausea, somnolence, and adverse effects was obtained. An EPDS was administered and a pill count to document the unused portion of their opioid prescription was recorded.

The primary outcome was opiate consumption in the acute postoperative period ( $24 \mathrm{hrs}, 48 \mathrm{hrs}$, and 1 week postoperatively). All pain medication received during that time frame were converted to morphine equivalents for statistical analysis using the standard table [17]. The secondary outcomes assessed included pain scores (visual analog scale) and adverse events or drug reactions. The statistical software package SPSS v23 (IBM Corp, Armonk, NY) was used for all data analysis. Dichotomous outcomes were compared utilizing chisquare testing and continuous variables were compared using t-tests. $p$ $<0.05$ was considered statistically significant. As this was a pilot study and at the time of design there were not any other trials administering 
gabapentin postoperatively, no a priori sample size calculation was performed. Based on other trial sample sizes we attempted to recruit 30 women to the pilot trial. The trial was stopped due to difficulty recruiting (due to excluding women planning any breastfeeding) and resident graduation.

\section{Results}

From October 2015-January 2017, 327 women were screened, 33 were found eligible for the study, and 13 women were consented and randomized as follows: 3 to gabapentin, 10 to placebo (Figure 1). One woman in the placebo group was withdrawn after having to be converted to general anesthesia during her cesarean. Complete follow up data were unable to be obtained on 6 participants because they were unavailable for contact one week after cesarean delivery. All 6 were randomized to the placebo group. However, in-hospital data were collected on all subjects.
Baseline characteristics including age, race, parity, gestational age, body mass index, length of cesarean delivery, infant birth weight were similar across the two groups (Table 1). Five women ( 2 gabapentin, 3 placebo) underwent a cesarean delivery with bilateral tubal ligation with no statistically significant difference found these between groups $(p=0.52)$. All cesareans were elective repeat procedures. Seven female and six male newborns were delivered.

There was a significant reduction in total morphine equivalent consumption during the first 48 hours after the cesarean (placebo vs. gabapentin: total during first 48 hours-( $22.6 \pm 14.1$ vs. $8.9 \pm 2.5, p=0.02$, Table 2). The mean pain scores at rest and with movement at 24,48 , and 1 week postoperatively had no statistically significant differences (Figure 2). Mean pain scores rated immediately before and between 2-4 hours after each dose of study drug were obtained. The differences in mean pain scores were significantly lower for women receiving gabapentin after the $5^{\text {th }}$ and $6^{\text {th }}$ dose $(p=0.008$ and 0.004 , respectively),

Table 1. Baseline characteristics of trial participants

\begin{tabular}{|c|c|c|c|c|}
\hline Variable & Overall population & Placebo $(n=10)$ & Gabapentin $(n=3)$ & $p$ value \\
\hline Age (yrs) & $31.3 / 32(5.5)$ & $30.1(5.1)$ & $35.3(5.5)$ & 0.16 \\
\hline EGA at delivery (wks) & $38.5 / 39.0(1.8)$ & $38.3(1.7)$ & $37.3(1.8)$ & 0.21 \\
\hline Parity & $2.3 / 2.0(1.1)$ & $2.3(1.2)$ & $2.3(0.6)$ & 0.97 \\
\hline BMI & $38.1 / 3401(11.3)$ & $39.6(12.5)$ & $33.3(4.4)$ & 0.42 \\
\hline Length of CD (mins) & $85.2 / 78.5(28.5)$ & $84.6(30.8)$ & $87.3(26.1)$ & 0.89 \\
\hline Birthweight (g) & $3287 / 3504(663)$ & $3276(691)$ & 3325 (698) & 0.92 \\
\hline $\begin{array}{c}\text { Racial/Ethnic group } \\
\text { African American } \\
\text { White } \\
\text { Mixed } \\
\text { Hispanic }\end{array}$ & $\begin{array}{l}6 \\
4 \\
1 \\
2\end{array}$ & $\begin{array}{l}4 \\
4 \\
1 \\
1\end{array}$ & $\begin{array}{l}2 \\
0 \\
0 \\
1\end{array}$ & 0.44 \\
\hline
\end{tabular}

BMI: body mass index; EGA: estimated gestational age; CD: cesarean delivery

Data are presented as mean/median (Standard Deviation), except for the racial/ethnic group which are the n of women in that category. Comparisons between groups by t-testing for means and chi-square testing for racial/ethnic distribution.

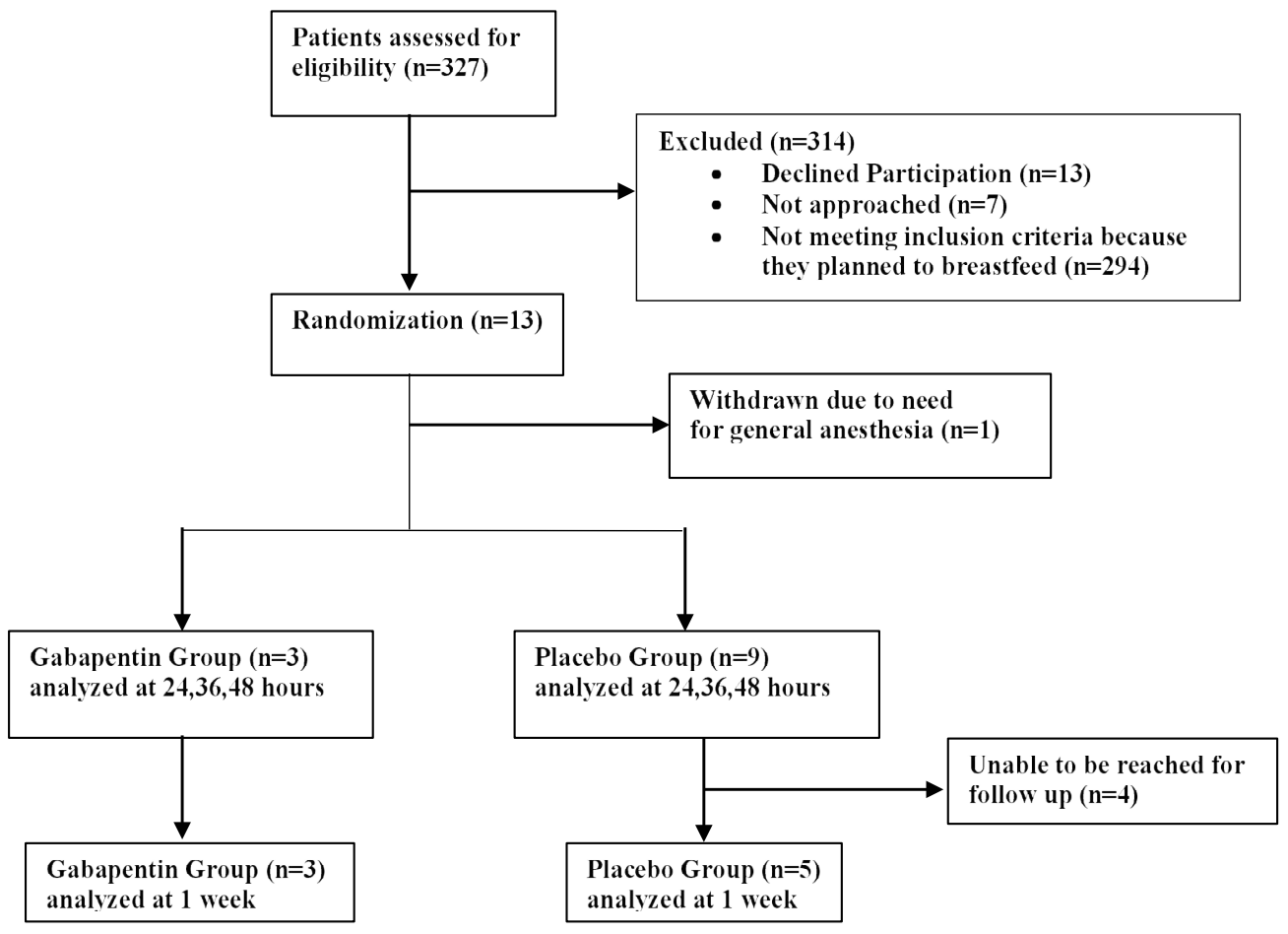

Figure 1. Trial profile for participant recruitment and analysis 


\section{Visual Analog Scale Pain Scores}

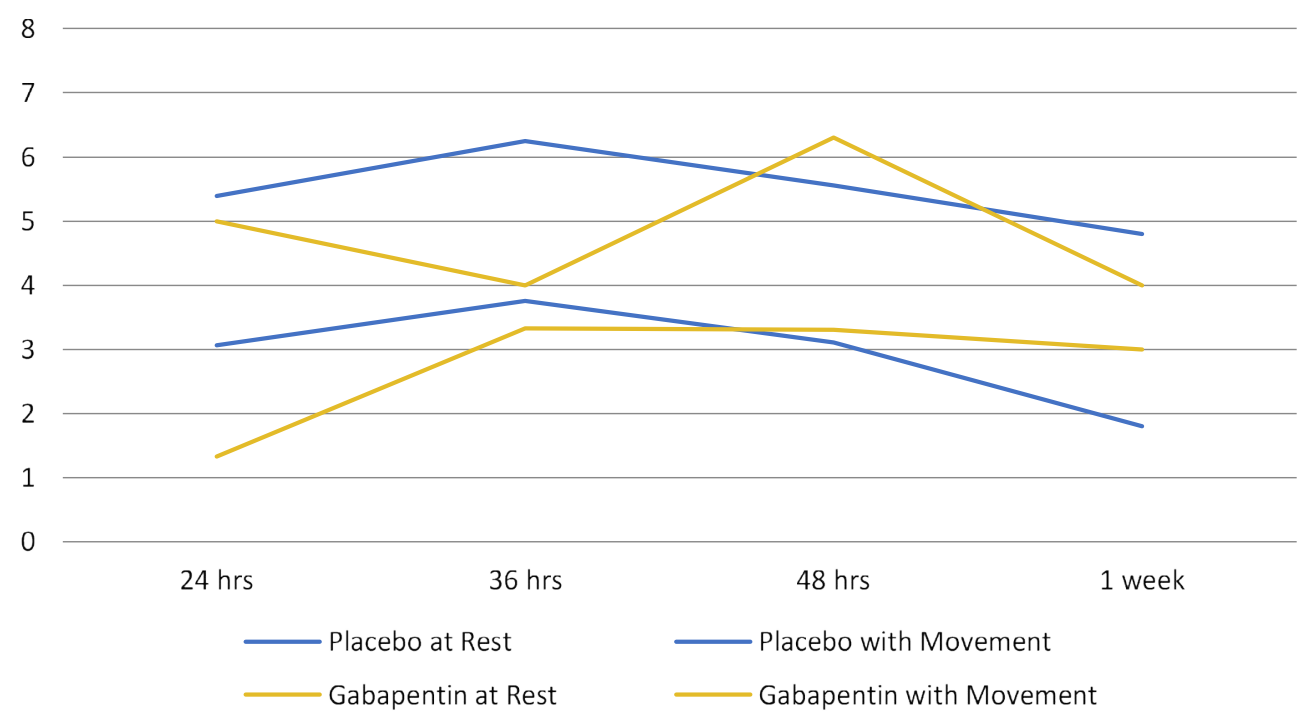

Figure 2. Mean visual analog scale scores on movement and at rest for gabapentin and placebo groups at each postoperative assessment. No significant difference was seen at any time point between the groups

Table 2. Opiate Consumption in Morphine Equivalents for each group

\begin{tabular}{|l|c|c|c|}
\hline Variable & $\begin{array}{c}\text { Placebo } \\
(\mathbf{n = 9})\end{array}$ & $\begin{array}{c}\text { Gabapentin } \\
(\mathbf{n}=\mathbf{3})\end{array}$ & p value \\
\hline Mean morphine equivalents taken in $1^{\text {st }} 24 \mathrm{hrs}$ & $11.4(11.5)$ & $2.2(1.9)$ & 0.21 \\
\hline Mean morphine equivalents taken in 2nd $24 \mathrm{hrs}$ & $11.2(6.8)$ & $6.7(1.7)$ & 0.30 \\
\hline $\begin{array}{l}\text { Mean total morphine equivalents taken at } 48 \\
\text { hours after cesarean }\end{array}$ & $22.6(14.1)$ & $8.9(2.5)$ & 0.02 \\
\hline $\begin{array}{l}\text { Total number of pills taken in 1 week after } \\
\text { discharge- mean }\end{array}$ & $23(19)$ & $15(16)$ & 0.56 \\
\hline
\end{tabular}

Data are presented as mean (SD). Comparisons made by t-testing.

Table 3. Adverse effects

\begin{tabular}{|l|c|c|c|}
\hline Outcome/side effect & Placebo & Gabapentin & p value \\
\hline Any moderate or worse side effect at 24 hours & $4(50 \%)$ & $2(66 \%)$ & 0.62 \\
\hline Any moderate or worse side effect at 36 hours & $2(29 \%)$ & $1(100 \%)$ & 0.37 \\
\hline Any moderate or worse side effect at 48 hours & $1(11 \%)$ & $0(0 \%)$ & 0.55 \\
\hline EPDS at consent & $1.8(1.5)$ & $1.3(1.2)$ & 0.63 \\
\hline EPDS at 48 hours & $1.6(2.1)$ & $1.7(1.5)$ & 0.98 \\
\hline EPDS at 1 week & $0.8(0.8)$ & $1.7(1.5)$ & 0.33 \\
\hline
\end{tabular}

EPDS: Edinburgh Perinatal Depression Score

mainly due to the fact that all 3 women taking gabapentin rated their pain as a 0 at those time points (complete data not shown).

The most common rated moderate side effects were sleepiness, dizziness, and itching. However, there were no differences in the side effects experienced between the two groups (Table 3). Additionally, only one participant (placebo group) took any medication for any of the side effects at any time point. EPDS scores were also not different between the groups and no woman ever scored $>6$ on the scale.

At the one-week follow-up contact, all women in the gabapentin group stated that the pain control was improved with the study drug compared to their last cesarean $(100 \%$ vs. $50 \%$ in placebo group, $p=0.43$ ). When asked if they had another cesarean, they would take the drug they were given again, all women assigned to gabapentin said they would, compared to $50 \%$ of the women in the placebo group $(p=0.43)$.

\section{Discussion}

We demonstrated that in non-breastfeeding women, a postoperative course of gabapentin may reduce the opioid use in the early postcesarean period. This is important in light of the growing epidemic of opioid use in the United States. [1,18]

One of the main concerns for women undergoing a cesarean delivery is pain and how it will affect their ability to care for their families [14]. Therefore, discovering ways to enhance recovery after cesarean deliveries can have a great health impact. Gabapentin is effective at reducing postoperative opiate requirements and postoperative pain $[7,9,10,19]$. Most studies, including those evaluating gabapentin's effect on postoperative cesarean section pain, were designed for patients to receive a preoperative dose of gabapentin prior to incision [13-15,20]. The motivation for timing preoperatively is based on the theory that gabapentin inhibits pain transmission through reducing central sensitization after tissue damage $[7,8]$. However, the mechanism of postoperative pain involves numerous neurophysiologic and neurochemical mechanisms, and thus postoperative dosing of gabapentin has also been studied and shown to be beneficial at reducing postoperative pain $[8,9,16]$. To our knowledge, this is the first study evaluating the effect of moderately dosed gabapentin (600 mg) administered only in the postoperative period after cesarean delivery. One trial administered a single dose of $600 \mathrm{mg}$ preoperatively followed by $200 \mathrm{mg}$ every 8 hours for 2 days postoperatively [13]. That study noted a very small difference in pain scores but no difference in opiate consumption. This may have been due to the lower dose used postoperatively in that study. They did note improved satisfaction with women using gabapentin postoperatively.

While one meta-analysis and trial sequential analysis did not support the use of gabapentin in general post-operative pain management in surgery [21], a more recent one with more trials included noted that gabapentin prior to cesarean leads to lower VAS pain scores with movement at 24 hours and higher pain control satisfaction with no difference in maternal or neonatal side effects [20]. Gabapentin has been effectively incorporated into post-hysterectomy Enhanced Recovery After Surgery protocols $[19,22]$. 
Even with a small study number, the results of our pilot study suggest that the addition of postoperative gabapentin $(600 \mathrm{mg}$ every 8hours for the first 48hours) to the current multimodal treatment regimen for controlling postoperative cesarean pain may decrease opiate consumption. This should be verified in a larger trial using these doses. While our study did not demonstrate a difference in pain satisfaction scores between the two groups, all women in the gabapentin group said it enhanced their pain control compared to prior cesareans and that they would take it again for a future cesarean. In addition, pain scores after the $5^{\text {th }}$ and $6^{\text {th }}$ doses were rated at " 0 " on the visual analog scale by all women in the gabapentin group, significantly lower than women in the placebo group. While overall pain scores at rest and with movement showed no clear differences, it is possible that with the use of gabapentin, less morphine equivalents were required to achieve the same satisfaction in pain control. Additionally, the incidence of worrisome adverse side effects was minimal and no different between our study groups. It appears that in this small study, gabapentin is not only effective but also safe for mothers who need to be able to take care of both themselves and their newborns in the acute postoperative period. While the overall data may not currently support adding gabapentin to enhanced cesarean recovery recommendations at this time, this may be due to limited trials specifically looking at women after cesarean delivery [23].

Given the results of this study, further studies are warranted to evaluate the role of gabapentin in the multimodal pain regimen to control postoperative cesarean delivery pain. A larger trial focusing on post-cesarean use of gabapentin should be undertaken to confirm our pilot trial findings. Additionally, robust data on the transfer of gabapentin into breastmilk and the reporting any neonatal effects will be key areas of study moving forward. Currently, there are limited data on gabapentin's transfer into breastmilk, however the $\mathrm{NIH}$ LACTMED report notes that there were low levels in infant serum and that gabapentin is an "acceptable choice" for use during lactation [24]. Research on its use in breastfeeding women, which was not reported in other trials, is needed to evaluate for any adverse neonatal effects to discern between these effects from gabapentin or from increased opioid exposure. In addition, it would be interesting to look at the use of gabapentin in chronic pain/chronic opiate users as these are the women who are most at risk to fail traditional postoperative pain regimens and may gain the most benefit from the adjunctive effects of gabapentin.

A strength of our study was its triple blinded execution and centrally controlled randomization and allocation. In these ways we attempted to limit the risk of bias to our results. We utilized a standard randomization scheme which yielded uneven group allocation. Given more recruitment, these would likely have balanced out. Future trials should use block randomization to avoid this.

An obvious limitation to our study is the small study population and inability to perform subgroup analysis. There was an extremely low recruitment rate. This was largely due to our IRB requiring the exclusion of all breastfeeding mothers because at the time there was very limited safety data on infant exposure to gabapentin through breast milk. The Monks et. al. trial which used postoperative gabapentin in low doses and included breastfeeding women reported no difference in breastfeeding difficulties in women taking gabapentin and placebo [13]. Therefore, it would seem worthwhile to duplicate this pilot study in a larger, pragmatic population that included breastfeeding women utilizing the $600 \mathrm{mg}$ dose. Additionally, the uneven group distribution from our randomization scheme was a limitation. Future studies will use block randomization to overcome this limitation.

\section{Conclusion}

In summary, this small pilot study demonstrates that $600 \mathrm{mg}$ of gabapentin administered every 8 hours in the first 48 hours following a scheduled repeat cesarean delivery may decrease opiate consumption without significantly altering pain satisfaction or incidence of adverse side effects. While opiates are still necessary and have a long track record in postoperative obstetric pain, given the current epidemic of opiate abuse and addiction among reproductive age women, exploring ways to reduce opiate consumption may have a long-term health impact on this vulnerable population.

\section{Declarations}

Ethics approval and consent to participate- The trial was approved by the IUPUI Human Subjects Institutional Review Board prior to all study activities. All women provided written informed consent to participate in the trial.

Availability of data and material- De-identified trial data will be made available to investigators performing meta-analyses.

\section{Funding}

This trial was internally funded entirely by the Department of Obstetrics and Gynecology, IU School of Medicine. Part of Dr. Haas's time is funded by NIH grants for work in pharmacology in pregnancy.

\section{Authors' contributions}

All authors contributed to the development and implementation of this trial; TR, CE, DMH were primarily responsible for the analysis; all authors participated in manuscript presentation and approval of submission.

\section{Acknowledgement}

These results were presented as an oral presentation at the Central Association of Obstetricians and Gynecologists Annual Meeting in October 2016 in Las Vegas, NV. They were also presented at the IUSM Research Day June 2016.

Funding- Department of OB/GYN, IUSM internal grant

Financial Disclosure: The authors declare no conflicts of interest

Trial Registry: clinicaltrials.gov NCT\#02490345 (https://clinicaltrials. gov/ct2/show/NCT02490345)

Date of registration: 7/3/2015

Date of initial participant enrollment: October 2015.

\section{References}

1. Jones GH, Bruera E, Abdi S, Kantarjian HM (2018) The opioid epidemic in the United States-Overview, origins, and potential solutions. Cancer 124: 4279-4286. [Crossref]

2. American College of Obstetricians and Gynecologists (2014) ACOG Committee Opinion No. 524: Opioid abuse, dependence, and addiction in pregnancy. Obstet Gynecol 119: 1070-1076. [Crossref]

3. Maeda A, Bateman BT, Clancy CR, Creanga AA, Leffert LR (2014) Opioid abuse and dependence during pregnancy: temporal trends and obstetrical outcomes. Anesthesiology 121: 1158-1165. [Crossref]

4. Martin JA, Hamilton BE, Osterman M, Driscoll AK, Drake P (2018) Births: final data for 2016 Natl Vital Stat Rep 67: 1-55. [Crossref]

5. Landau R, Bollag L, Ortner C (2013) Chronic pain after childbirth. Int J Obstet Anesth 22: 133-145. [Crossref]

6. Eisenach JC, Pan PH, Smiley R, Lavand'homme P, Landau R, et al. (2008) Severity 
of acute pain after childbirth, but not type of delivery, predicts persistent pain and postpartum depression. Pain 140: 87-94. [Crossref]

7. Chang CY, Challa CK, Shah J, Eloy JD (2014) Gabapentin in acute postoperative pain management. Biomed Res Int 2014: 631756. [Crossref]

8. Schmidt PC, Ruchelli G, Mackey SC, Carroll IR (2013) Perioperative gabapentinoids: choice of agent, dose, timing, and effects on chronic postsurgical pain. Anesthesiology 119: 1215-1221. [Crossref]

9. Clarke H, Bonin RP, Orser BA, Englesakis M, Wijeysundera DN, et al. (2012) The prevention of chronic postsurgical pain using gabapentin and pregabalin: a combined systematic review and meta-analysis. Anesth Analg 115: 428-442. [Crossref]

10. Peng PW, Wijeysundera DN, Li CC (2007) Use of gabapentin for perioperative pain control -- a meta-analysis. Pain Res Manag 12: 85-92. [Crossref]

11. Hurley RW, Cohen SP, Williams KA, Rowlingson AJ, Wu CL (2006) The analgesic effects of perioperative gabapentin on postoperative pain: a meta-analysis. Reg Anesth Pain Med 31: 237-247. [Crossref]

12. Pandey CK, Singhal V, Kumar M, Lakra A, Ranjan R, et al. (2005) Gabapentin provides effective postoperative analgesia whether administered pre-emptively or postincision. Can J Anaesth 52: 827-831. [Crossref]

13. Monks DT, Hoppe DW, Downey K, Shah V, Bernstein P, et al. (2015) A Perioperative Course of Gabapentin Does Not Produce a Clinically Meaningful Improvement in Analgesia after Cesarean Delivery: A Randomized Controlled Trial. Anesthesiology 123: 320-326. [Crossref]

14. Moore A, Costello J, Wieczorek P, Shah V, Taddio A, et al. (2011) Gabapentin improves post cesarean delivery pain management: a randomized, placebo-controlled trial. Anesth Analg 112: 167-173. [Crossref]

15. Short J, Downey K, Bernstein P, Shah V, Carvalho JC (2012) A single preoperative dose of gabapentin does not improve post cesarean delivery pain management: a randomized, double-blind, placebo-controlled dose-finding trial. Anesth Analg 115: 1336-1342. [Crossref]
16. Pandey CK, Navkar DV, Giri PJ, Raza M, Behari S, et al. (2005) Evaluation of the optimal preemptive dose of gabapentin for postoperative pain relief after lumbar discectomy: a randomized, double-blind, placebo-controlled study. $J$ Neurosurg Anesthesiol 17: 65-68. [Crossref]

17. Knotkova H, Fine PG, Portenoy RK (2009) Opioid rotation: the science and the limitations of the equianalgesic dose table. J Pain Symptom Manage 38: 426-439. [Crossref]

18. Epstein RA, Bobo WV, Martin PR, Morrow JA, Wang W, et al. (2013) Increasing pregnancy-related use of prescribed opioid analgesics. Ann Epidemiol 23: 498-503. [Crossref]

19. Alayed N, Alghanaim N, Tan X, Tulandi T (2014) Preemptive use of gabapentin in abdominal hysterectomy: a systematic review and meta-analysis. Obstet Gynecol 123 1221-1229. [Crossref]

20. Felder L, Saccone G, Scuotto S, Monks DT, Carvalho JCA, et al. (2019) Perioperative gabapentin and post cesarean pain control: A systematic review and meta-analysis of randomized controlled trials. Eur J Obstet Gynecol Reprod Biol 233: 98-106. [Crossref]

21. Fabritius ML, Geisler A, Petersen PL, Nikolajsen L, Hansen MS, et al. (2016) Gabapentin for post-operative pain management - a systematic review with metaanalyses and trial sequential analyses. Acta Anaesthesiol Scand 60: 1188-1208. [Crossref]

22. Nelson G, Altman AD, Nick A, Meyer LA, Ramirez PT, et al. (2016) Guidelines for postoperative care in gynecologic/oncology surgery: Enhanced Recovery After Surgery (ERAS(R)) Society recommendations--Part II. Gynecol Oncol 140: 323-332. [Crossref]

23. Wilson RD, Caughey AB, Wood SL, Macones GA, Wrench IJ, et al. (2018) Guidelines for Antenatal and Preoperative care in Cesarean Delivery: Enhanced Recovery After Surgery Society Recommendations (Part 1). Am J Obstet Gynecol 219: 523 e521-523 e15. [Crossref]

24. LACTMED: Gabapentin- CASRN: 60142-96-3 Available at www.toxnet.nlm.nih.gov

Copyright: (2019 Randolf T. This is an open-access article distributed under the terms of the Creative Commons Attribution License, which permits unrestricted use, distribution, and reproduction in any medium, provided the original author and source are credited. 